\title{
Informative SSR markers for genotyping and intervarietal differentiation of cultivated oat
}

\author{
Dubovets N.I.*, Sycheva E.A., Sokolyuk A.V., Bondarevich E.B., Solovey L.A. \\ Institute of Genetics and Cytology NAS of Belarus, Minsk, Belarus \\ *email: N.I.Dubovets@igc.by
}

Currently, DNA technologies occupy a leading position in the breeding programs of developed countries, which makes it possible to speed up significantly the process of creating new varieties. Among the DNA markers used in this case, SSR markers are the most in demand, especially at the stage of studying the genetic diversity of breeding material. Taking into account the fact that oat breeding in Belarus is carried out exclusively by traditional methods, we set a goal to form a set of informative SSR markers for genotyping Avena sativa L. 14 markers were selected based on the analysis of literature data, which were tested on 22 oat varieties. Half of them were Belarusian varieties, among the rest -3 varieties of European selection, 4 varieties of the Baltic selection and 4 varieties of the Russian selection. An indicator such as the value of informational polymorphism (PIC) was used to assess the effectiveness of the used marker system. Primer pairs to two monomorphic loci $A M 15$ and $A M 38$ had a zero PIC value in all studied cultivars, and only one pair of primers to the $A M 83$ locus, which revealed 2 alleles, was moderately informative. The remaining 11 markers were highly informative according to the classification of Botstein et al. (1980) with PIC variation from 0.57 to 0.93 . All of them are included in the set for the study of genetic variability of oat cultivars. Of these, 7 markers (AM4, AM5, AM7, AM11, AM14, AM22, and AM30) with a PIC in the range from 0.82 to 0.93 are recommended for differentiation of closely related genotypes and varieties certification. The use of the created set of markers will make it possible to assess the level of genetic variability of the varietal gene pool involved in the breeding process on the territory of Belarus, to determine the degree of genotype relationships, and, as a result, to more efficiently select parental pairs during hybridization. 